TITLE:

\title{
Spontaneous rhythmic motion of a polymer chain in a continuous- wave laser field
}

\section{AUTHOR(S):}

Kitahata, H; Mayama, H; Yoshikawa, K

\section{CITATION:}

Kitahata, H ... [et al]. Spontaneous rhythmic motion of a polymer chain in a continuous-wave laser field. Physical Review E 2004, 70(2): 021910.

\section{ISSUE DATE:}

2004-08

URL:

http://hdl.handle.net/2433/49858

RIGHT:

Copyright 2004 American Physical Society 
PHYSICAL REVIEW E 70, 021910 (2004)

\title{
Spontaneous rhythmic motion of a polymer chain in a continuous-wave laser field
}

\author{
Hiroyuki Kitahata, Hiroyuki Mayama,* and Kenichi Yoshikawa ${ }^{\dagger}$ \\ Department of Physics, Graduate School of Science, Kyoto University, and CREST, Kyoto 606-8502, Japan
}

(Received 26 February 2004; published 24 August 2004)

\begin{abstract}
Recently, it has been found that a giant DNA molecule exhibits a repetitive change in conformation between an elongated coil and folded compact states under irradiation by a continuous IR laser. We report here a theoretical model to explain such spontaneous rhythmic motion. The generation of the rhythm can be attributed to the competition between optical attraction in the compact state and the instability of the compact state at the laser focus due to heating. This rhythmic conformational change is discussed in relation to the working mechanism of biological molecular motors.
\end{abstract}

DOI: 10.1103/PhysRevE.70.021910

PACS number(s): 87.14.Gg, 82.40.Bj, 05.70.Ln

\section{INTRODUCTION}

The best known macroscopic engine utilizes the Carnot cycle, which employs both isothermal and adiabatic processes. Unfortunately, the Carnot cycle cannot be used to explain biological molecular motors. For molecular machinery on a scale of $100 \mathrm{~nm}$ in an aqueous solution, the characteristic time for thermal diffusion is on the order of $100 \mathrm{~ns}$. However, molecular motors work at frequencies of less than $1 \mathrm{kHz}$ [1-4], and therefore the duration of various steps is greater than $1 \mathrm{~ms}$, allowing time for heat to diffuse in and making the process nonadiabatic. Thus, the Carnot cycle cannot be used to explain molecular machinery and design micromachines.

Recently, it was found that under stationary irradiation by a focused IR laser beam (wavelength $\lambda=1064 \mathrm{~nm}$ ), a single DNA molecule undergoes a repetitive change in conformation between an elongated coil and folded compact states [5]. This preliminary observation on the repetitive conformational change has been interpreted superficially as the interplay between different effects of a focused laser beam, namely the attractive optical potential and local heating due to the absorption of photons at the laser focus. In this paper, we propose a theoretical model to interpret such a rhythmic oscillation arising from a discrete conformational change in a single polymer chain under thermodynamically open conditions of heat exchange with the solvent.

\section{EXPERIMENTS}

Samples were prepared as follows: $0.3 \mu \mathrm{M}$ T4 DNA in nucleotides (Nippon Gene, $166 \mathrm{kbp}$ ) was dispersed into a buffer solution (10 mM Tris- $\mathrm{HCl}, \mathrm{pH}$ 7.4) that included $0.9 \mu \mathrm{M}$ fluorescence dye, 4', 6-Diamidino-2-phenylindole dihydrochloride (DAPI) (Molecular Probes Co., Ltd.), $73 \mathrm{mg} \mathrm{mL}^{-1}$ poly(ethylene glycol) (PEG) (average $M_{r}=2$

\footnotetext{
*Present address: Nanotechonology Research Centre, Research Institute for Electronic Science, Hokkaido University, Sapporo 0010021, Japan.

${ }^{\dagger}$ Author to whom correspondence should be addressed. Electronic address: yoshikaw@scphys.kyoto-u.ac.jp
}

$\times 10^{4}$, Kishida Chemical), $4 \%$ (v/v) 2-mercaptethanol (Nacalai Tesque), and $0.3 \mathrm{M} \mathrm{KCl}$ (Wako). The prepared samples were allowed to stand at ambient temperature, $20 \pm 1^{\circ} \mathrm{C}$, for $30 \mathrm{~min}$ before observation. Under these conditions, individual DNA molecules undergo a discrete transition from compact to elongated conformations with an increase in temperature [6]. The DNA solution was observed with an inverted fluorescence microscope (Nikon TE-300) equipped with an objective lens [Nikon Plan Fluor, 100, numerical aperture (NA) 1.30], and a high-sensivity silicon intensifier target (SIT) camera (Hamamatsu Photonics) and optical tweezers (continuous-wave Nd:YAG laser, Spectron, SL902T, wavelength $\lambda=1064 \mathrm{~nm}$ ). Under fluorescence microscopy, it is known that folded DNA is observed as an optical dot and can be trapped by a focused laser [7]. We observed the time-dependent change of the fluorescence image of a single T4 DNA under continuous irradiation of the laser beam.

Figure 1(a) exemplifies oscillation of a single T4 DNA: A bright spot at the laser focus becomes shallow, and then turns back to a bright spot. Such a rhythmic change continues with an interval of $\sim 2 \mathrm{~s}$. Based on this single-molecule observation, a schematic representation of the rhythmic conformational change in a single DNA molecule is shown in Fig. 1(b). A folded DNA molocule is trapped and heated at the laser focus, where a temperature gradient is induced around the laser focus due to the weak photoabsorption on the third harmonics of O-H stretching. It is estimated that the temperature at the focus of the laser beam is $\sim 5 \mathrm{~K}$ higher than the environment [8].

Figure 2(a) shows time courses of the fluorescence intensity and position of the center of mass in the rhythmic conformational change at laser power $=1000 \mathrm{~mW}$ : the fluorescence intensity changes periodically between high and low intensity with large fluctuation. Thus, the rhythmic conformational change in a single DNA molecule can be characterized as the periodic transition between a bright spot and a blurred swollen image. The time course of the position of the center of mass indicates that a DNA molecule exhibits periodic motion between the center of the laser focus and a "cool" ambient area. In the autocorrelation, the main peak appears at around $1 \mathrm{~Hz}$. Figure 2(b) shows the trajectory on the two-variable plane. 
(a)

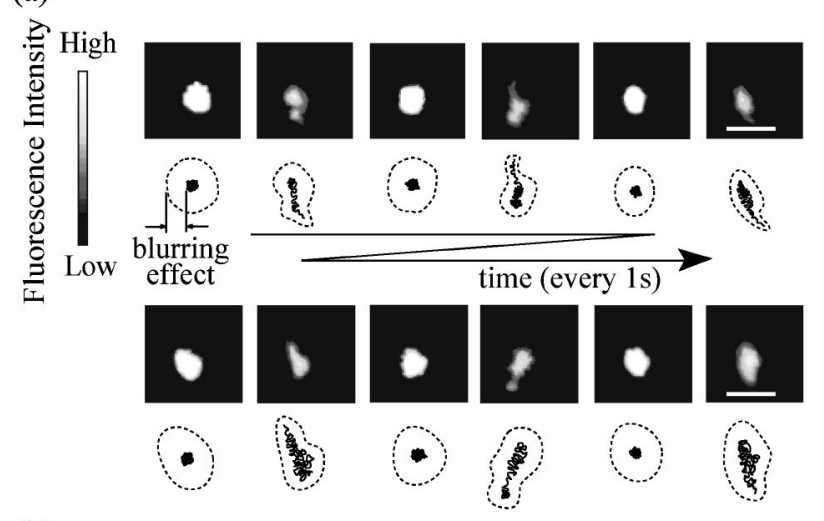

(b)

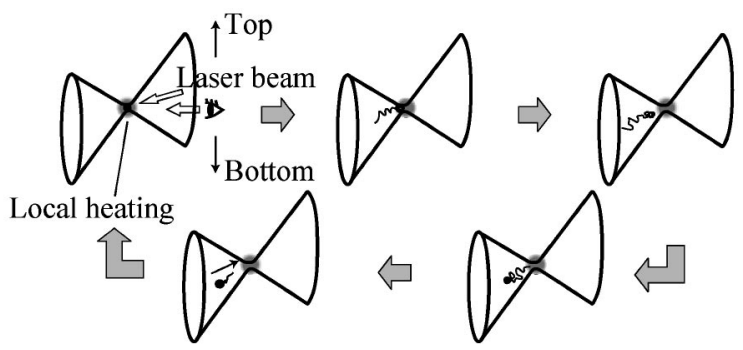

FIG. 1. (a) Upper: snapshots of fluorescence images of the rhythmic conformational change in a single DNA molecule under irradiation by optical tweezers (laser power: $1000 \mathrm{~mW}$, time interval: $1 \mathrm{~s}$ ). The bar corresponds to $1 \mu \mathrm{m}$. Lower: schematic representation of the conformation of a DNA molecule with a blurring effect. (b) Schematic representation of rhythmic conformational change in a single DNA molecule under irradiation by a focused cw laser beam. A folded DNA molecule is attracted to the laser focus and heated. Then it becomes unfolded, receives less attractive force, and moves away from the focus driven by Brownian motion. The DNA molecule out of the focus feels the ambient unheated circumstance and returns back to the folded state. The folded DNA is again attracted to the focus, and this cycle repeats.

\section{DISCUSSION}

Before discussing the detailed mechanism of the oscillatory change in the conformation of a single giant DNA molecule, we will briefly address the thermodynamic properties of a single semiflexible molecular chain. It has become clear that, in general, a semiflexible polymer chain undergoes a discrete transition between an elongated coil and folded compact states [9]. Since a single semiflexible chain constitutes a microscopic thermodynamic system with a finite number of elements, the free energy to describe the discrete transition is given by a double-minimum profile. Thus, the conformational transition in a single semiflexible chain corresponds to a first-order phase transition according to the criterion of Landau [10]. Reflecting the finiteness of the number of elements on individual molecular chains, there is a rather wide region of the coexistence between the elongated and compact states, and therefore the transition appears to be continuous with regard to the physico-chemical properties of an ensemble of chains, such as viscosity and light scattering [11].

We consider the mechanism of the oscillation of a single DNA molecule under irradiation by a cw laser beam from the (a)
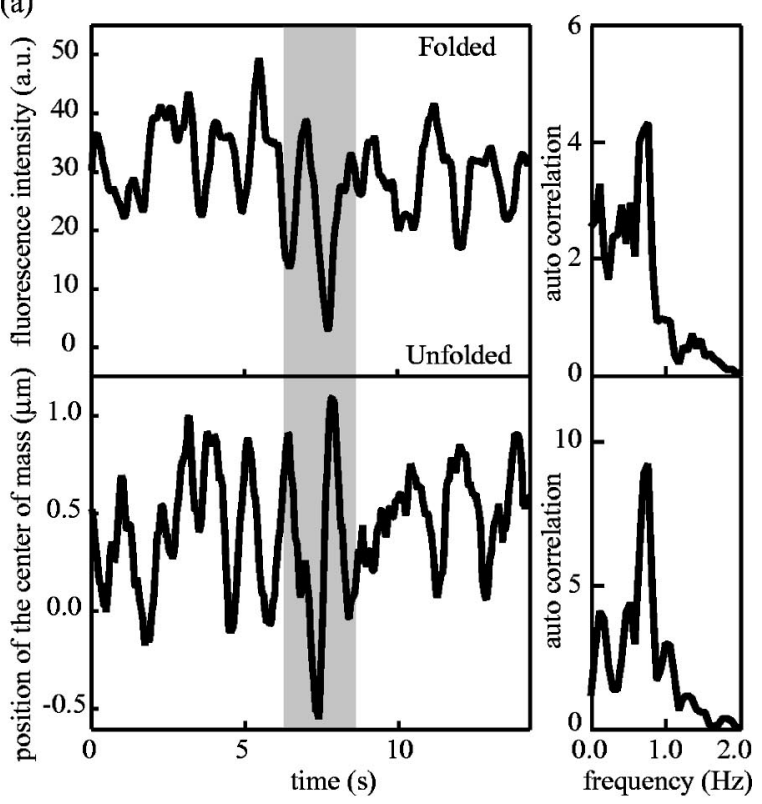

(b)

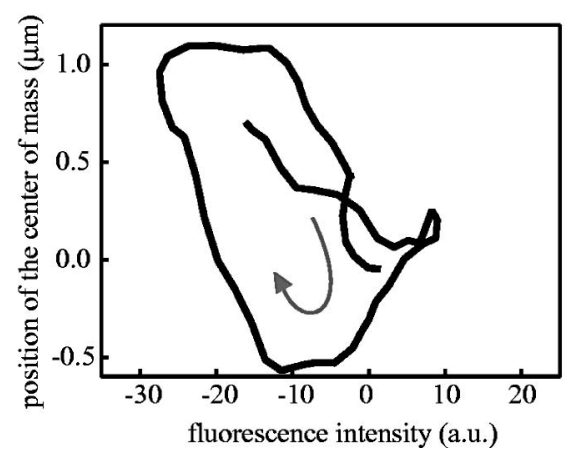

FIG. 2. (a) Left column: time trace of fluorescence intensity and the position of the center of mass obtained from fluorescence images. Right column: autocorrelation of the time traces of both variables. (b) The trajectory on the two-variable plane for the data in the shaded area in (a).

viewpoint of the free energy. The bimodality in the free energy, $F$, in a finite system implies that kinetic characteristics can be described by cubic relationships, as in Eq. (2), with respect to an order parameter, $\eta$, which corresponds to the density of the DNA molecule,

$$
\begin{gathered}
\epsilon \frac{\partial \eta}{\partial t} \simeq-\frac{\partial F}{\partial \eta}, \\
F=\alpha \eta^{2}(\eta-1)^{2}+\beta\left[T\left(r_{c}\right)-T_{0}\right] \eta,
\end{gathered}
$$

where $\epsilon$ is the proportionality constant, $r_{c}$ is the position of the center of the mass of a polymer chain, and $\alpha, \beta$, and $T_{0}$ are positive constants that determine the shape of the freeenergy profile.

For the profile of temperature, $T$, around the focused laser, 


$$
\frac{\partial T}{\partial t}=D \nabla^{2} T+G(r)
$$

where $G(r)$ corresponds to the energy supplied by the laser beam, which has a large value only near the focus, and $D$ is the thermal diffusion constant. In a three-dimensional system, the temperature profile near the focus is

$$
T=T_{\max } \exp \left(-\frac{r^{2}}{r_{\text {temp }}^{2}}\right),
$$

where $T_{\max }$ is the temperature at the focus, and $r_{\text {temp }}$ is the typical radius that relates to the diffusion constant, $D$, and the energy induced by the laser beam, $F\left(r_{\mathrm{c}}\right)$. Considering that the diameter of a DNA chain is $\sim 2 \mathrm{~nm}$, which is much smaller than the optical wavelength, the optical potential can be adequately described by the Rayleigh resume [12]. The time dependence of $r_{c}$ is

$$
\begin{aligned}
\frac{\partial r_{c}}{\partial t} & \simeq-\frac{\partial U}{\partial r_{c}}+f_{\text {scatt }}+\zeta(t), \\
U & \simeq-\eta U_{0} \frac{1}{r_{c}^{2}+r_{\text {trap }}^{2}},
\end{aligned}
$$

where $U$ is the optical potential induced by the focused laser beam, $f_{\text {scatt }}$ is the scattering force by the laser beam, $\zeta(t)$ is the Gaussian white noise, and $U_{0}$ and $r_{\text {trap }}$ are the constants that correspond to the depth and width of the optical potential, respectively. The scattering force $f_{\text {scatt }}$ is attributed not only to the direct effect of the laser force but also to an indirect convective effect via water.

In the above equations, the nature of the first-order phase transition between folded and unfolded states is incorporated in Eqs. (1) and (2), and the competition between trapping force and scattering force is depicted in Eq. (5). These two aspects are the sources of interaction between the density $\eta$ and the position of the center of mass $r_{c}$.

From a system with coupled differential equations, the temporal change in the conformation and position of a single chain can be numerically obtained as shown in Fig. 3(a). The density of the DNA molecule, $\eta$, shows a periodic change. The position of the center of mass of the DNA molecule also oscillates with some time delay with respect to $\eta$. These time traces correspond at least qualitatively to the experimental results shown in Fig. 2. The trajectory on the $\eta-r_{c}$ plane as depicted in Fig. 3 exhibits a similar feature to that in the experiment including the direction of rotation on the phase plane [see Fig. 2(b)]. It is thus concluded that our theoretical framework reproduces the essence of the experimental trends. As there remain many uncertain parameters in the equations at present, we do not try to perform the simulation by changing each parameter.

The theoretical framework, which is associated with the periodic conformational change of a polymer chain and spontaneous motion under nonequilibrium conditions, may

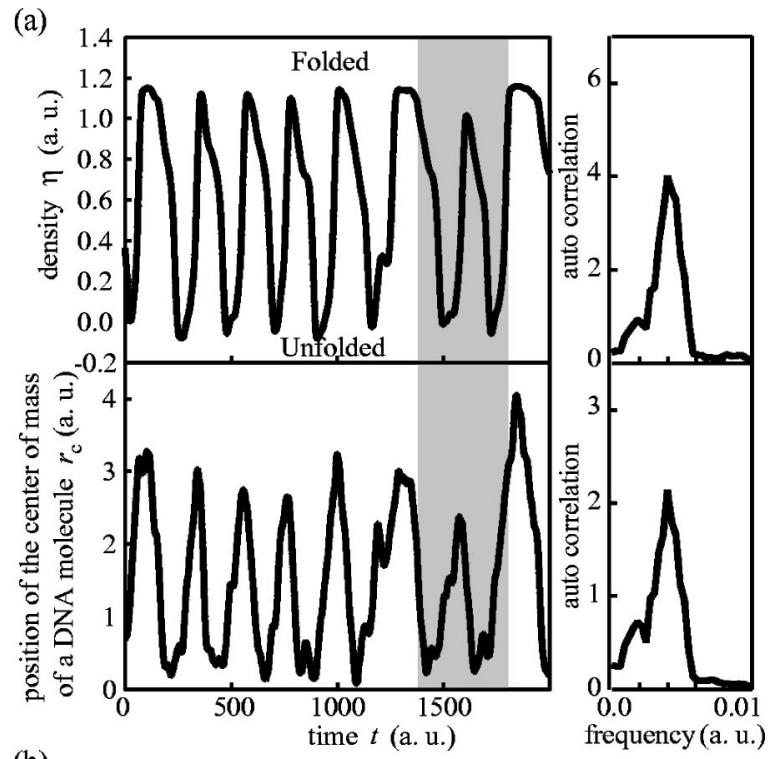

(b)

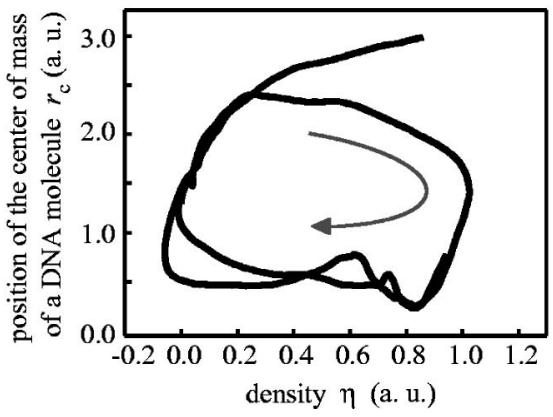

FIG. 3. Results of numerical calculations using Eqs. (1)-(6). (a) Left column: time trace of the order parameter, $\eta$, which corresponds to the density of the DNA molecule, and the position of the center of mass of the DNA molecule, $r_{c}$. Right column: autocorrelation of the time traces of $\eta$ and $r_{c}$. (b) The trajectory on the $\eta-r_{c}$ phase plane for the data in the shaded area in (a). The parameters used here are $\epsilon=10, \alpha=1, \beta=1, T_{0}=0.5, T_{\max }=0.76, r_{\text {temp }}=2.0$, $f_{\text {scatt }}=0.025, U_{0}=1.0$, and $r_{\text {trap }}=2.0$. The variance of Gaussian noise is 3. The initial state is $r_{c}=0.5$ and $\eta=0.5$.

be related to the working mechanism of molecular motors. It has been reported that the discrete conformational change in molecular motors under an ATP supply is closely related to the function of such motors, for instance myosin [13] and $\mathrm{F}_{1}$-ATPase $[4,14]$. The discrete conformational change in molecular motors driven by the ATP supply is expected to correspond to the first-order phase transition driven by a focused laser beam.

\section{CONCLUSION}

In conclusion, a numerical simulation showed that a single polymer chain can undergo a rhythmic conformational change under a microscopic temperature gradient. The essential features reported for the rhythmic change of a DNA 
chain under a focused IR laser were reproduced within the theoretical framework. Studies on self-excitation in such a rhythmic change in a microscopic system may be useful to obtain the deeper understanding of the mechanism of molecular motors in biological systems. Such studies may also aid in the development of a theoretical framework toward the design of micromotors for practical applications. The present study considered the phenomenon of self-motion without any physical load to generate work. Further study is needed to extend these findings to the realization of artificial microma- chinery on a macromolecule, especially focusing on the efficiency of energy conversion.

\section{ACKNOWLEDGMENTS}

The authors thank Masatoshi Ichikawa (Kyoto University, Japan) for the discussion on the mechanism. This work was supported in part by Grants-in-Aid for the 21st Century COE (Center for Diversity and Universality in Physics), and Scientific Research B.
[1] A. Katchalsky and P. F. Curran, Nonequilibrium Thermodynamics in Biophysics (Harvard University Press, Cambridge, 1965).

[2] R. P. Feynman, R. B. Leighton, and M. Sands, The Feynman Lectures on Physics (Addison-Wesley, Reading, MA, 1963), Vol. I.

[3] K. Yoshikawa and H. Noguchi, Chem. Phys. Lett. 303, 10 (1999).

[4] R. Yasuda, H. Noji, M. Yoshida, K. Kinoshita, Jr., and H. Itoh, Nature (London) 410, 898 (2001).

[5] H. Mayama, S. M. Nomura, H. Oana, and K. Yoshikawa, Chem. Phys. Lett. 330, 361 (2000); H. Mayama and K. Yoshikawa, Faraday Discuss. 120, 67 (2001).

[6] H. Mayama, T. Iwataki, and K. Yoshikawa, Chem. Phys. Lett. 318, 113 (2000).

[7] Y. Matsuzawa, K. Hirano, K. Mori, S. Katsura, K. Yoshikawa, and A. Mizuno, J. Am. Chem. Soc. 121, 11581 (1999).

[8] By assuming that the absorption constant of water is $0.1 \mathrm{~cm}^{-1}$ and that the heat conductivity of water is $0.6 \mathrm{~W} \mathrm{~m}^{-1} \mathrm{~K}^{-1}$, the rise in temperature at the focus is estimated to be $\sim 5 \mathrm{~K}$. See $\mathrm{S}$. Wurlitzer, C. Lautz, M. Liley, C. Duschl, and T. M. Fischer, J. Phys. Chem. B 105, 182 (2001).

[9] M. Ueda and K. Yoshikawa, Phys. Rev. Lett. 77, 2133 (1996); K. Yoshikawa, in Complexity and Diversity, edited by E. R. Nakamura, K. Kudo, O. Yamanaka, and Y. Tamagawa (Springer-Verlag, Berlin, 1997).

[10] L. D. Landau and E. M. Lifshitz, Statistical Physics, 3rd ed. (Pergamon, Oxford, 1980).

[11] V. A. Bloomfield, Curr. Opin. Struct. Biol. 6, 334 (1996).

[12] K. Svoboda and S. M. Block, Annu. Rev. Biophys. Biomol. Struct. 23, 247 (1994).

[13] Y. Suzuki, T. Yasunaga, R. Ohkura, T. Wakabayashi, and K. Sutoh, Nature (London) 396, 380 (1998).

[14] P. D. Boyer, Biochim. Biophys. Acta Bioenerg. 1458, 252 (2000). 